\title{
C-reactive protein levels predict systolic heart failure and outcome in patients with first ST-elevation myocardial infarction treated with coronary angioplasty
}

Christian Stumpf ${ }^{1,2}$, Ahmed Sheriff ${ }^{3}$, Stefan Zimmermann ${ }^{2}$, Liane Schaefauer ${ }^{1}$, Christian Schlundt ${ }^{1}$, Dorette Raaz ${ }^{1}$, Christoph D. Garlichs ${ }^{4}$, Stephan Achenbach ${ }^{1}$

\begin{abstract}
${ }^{1}$ Department of Cardiology, University of Erlangen-Nuremberg, Erlangen, Germany ${ }^{2}$ Department of Cardiology, Klinikum Bayreuth, Germany

${ }^{3}$ Department of Nephrology and Intensive Care Medicine, Charité Berlin, Germany ${ }^{4}$ Diakonissenkrankenhaus Flensburg, Germany
\end{abstract}

Submitted: 31 October 2016

Accepted: 15 February 2017

Arch Med Sci 2017; 13, 5: 1086-1093

DOI: https://doi.org/10.5114/aoms.2017.69327

Copyright $\odot 2017$ Termedia \& Banach

\section{Abstract}

Introduction: There is growing evidence that inflammation plays a pivotal role in the etiology and progression of atherosclerosis. High C-reactive protein (CRP) levels have been associated with high mortality in patients with acute myocardial infarction (AMI). Furthermore, in animal models CRP has been found to significantly increase infarct size. So there is growing evidence that CRP is not only a marker for cardiovascular disease but also might be pathogenic. The aim of our study was to test the hypothesis that peak CRP levels could predict heart failure (HF) in ST-elevation myocardial infarction (STEMI) patients.

Material and methods: Eighty-one consecutive patients with STEMI were prospectively enrolled in the study. C-reactive protein concentrations were measured on admission and after $6,12,24,30,48,72$ and $96 \mathrm{~h}$. We assessed the association between the elevation of CRP, heart failure and cardiovascular mortality following the first 12 months after STEMI.

Results: C-reactive protein levels reached a peak after $48 \mathrm{~h}$. Patients with STEMI and signs of HF showed significantly higher peak CRP levels. We found a positive correlation between maximum CK levels and peak CRP and a negative correlation between left ventricular ejection fraction (EF) and peak CRP. One year total mortality and HF mortality rates were found to be higher in patients with peak $C R P>47.5 \mathrm{mg} / \mathrm{l}$ than in those with CRP below that level $(p<0.001)$.

Conclusions: Peak CRP levels in STEMI patients predict emergence of HF. Peak CRP is also a strong predictor of global and cardiovascular mortality during the following year after STEMI.

Key words: C-reactive protein, heart failure, ST-elevation myocardial infarction, inflammation, clinical outcome.

\section{Introduction}

In the last decade there has been growing evidence that inflammation plays a pivotal role in the etiology and progression of atherosclerosis [1-4]. Increased levels of different markers of inflammation have been associated with either an increased risk of developing coronary artery disease (CAD) or with poor prognosis in patients with known CAD [5].

\author{
Corresponding author: \\ Christian Stumpf MD \\ Department of Cardiology \\ Medical Clinic 2 \\ University Clinic of Erlangen \\ Ulmenweg 18 \\ 91054 Erlangen, Germany \\ Fax: +49 32121082059 \\ E-mail: ch.stumpf@web.de
}


Amongst the inflammatory mediators, C-reactive protein (CRP) has emerged as an independent risk indicator. Observational studies have consistently reported that elevated CRP levels have clear prognostic value for major cardiovascular events and even mortality [6-8]. Although there is heterogeneity in the predictive value of CRP, the general consensus acknowledges a relation between CRP and cardiovascular disease. The impact of CRP on cardiovascular outcome has been emphasized by data showing that risk reduction for a first myocardial infarction seemed to be directly related to high sensitivity CRP (hsCRP) levels [9].

Furthermore, many prospective studies have demonstrated that CRP strongly and independently predicts adverse cardiovascular events, including myocardial infarction, ischemic stroke, and sudden cardiac death in individuals both with and without over CAD [10]. C-reactive protein is believed to be both a marker and a mediator of atherosclerosis and CAD. It plays a pivotal role in many aspects of atherogenesis including activation of the complement pathway, lipid uptake by macrophages, and release of proinflammatory cytokines, induces the expression of tissue factor in monocytes, promotes endothelial dysfunction and inhibits nitric oxide production.

As far back as in 1954, the value of estimating CRP levels as a measure of myocardial damage in the setting of acute myocardial infarction was already known [11]. Later on, CRP could be detected within and around necrotic myofibers in myocardial infarcts in rabbits. The CRP depositions have meanwhile also been found in human hearts during myocardial infarction in co-localization with activated complement [12]. Furthermore, it was shown that circulating CRP-complexes correlated significantly with the local concentration and the extent of the deposition of both CRP and complement. These observations are supported by animal studies in which human CRP injected into rats has been shown to increase infarct size by approximately $40 \%$, whereas in vivo complement depletion completely abrogated this effect [13]. Recently, a small-molecule inhibitor of CRP has been found in rats to attenuate the increase in infarct size and cardiac dysfunction in reaction to human CRP [14].

Another recent study showed that selective CRP apheresis in pigs significantly reduced the CRP levels. C-reactive protein apheresis after myocardial infarction was a safe and feasible therapeutic option for reduction of the size of the infarcted area and improved cardiac output as demonstrated by improved left ventricular ejection fraction (LVEF) [15].

Combining these clinical findings with experimental observations of the last years, a paradigm shift has occurred in which CRP is now no longer merely a marker, but is increasingly considered a mediator of cardiovascular disease $[13,16,17]$.

During acute myocardial infarction the incidence of heart failure and the strong influence of $\mathrm{HF}$ on mortality are among the most challenging problems to deal with in this clinical setting [18]. Pietila et al. found that patients who died of congestive heart failure following acute myocardial infarction (AMI) had significantly elevated CRP levels [19].

Therefore, the objective of our study was to evaluate the correlation between CRP levels and the risk of developing significant heart failure in patients with acute ST-elevation myocardial infarction (STEMI) and to test whether peak CRP levels could predict significant heart failure following STEMI.

\section{Material and methods}

\section{Patients}

Our study was designed as a single-center prospective observational cohort trial in the setting of first STEMI treated with primary percutaneous coronary intervention $(\mathrm{PCl})$. Eighty-one consecutive patients meeting the study inclusion and exclusion criteria were enrolled (Table I).

Inclusion criteria were as follows: typical stenocardial chest pain of at least 30 min duration; onset of symptoms $<12 \mathrm{~h}$ before hospital admission and electrocardiographic features of acute $\mathrm{MI}$ with STEMI $\geq 0.1 \mathrm{mV}$ in 2 or more contiguous limb leads or $\geq 0.2 \mathrm{mV}$ in 2 or more contiguous precordial leads and no history of previous myocardial infarction.

The exclusion criteria were lack of informed consent; prior coronary revascularization; prior myocardial infarction; cardiogenic shock; pre-existing congestive heart failure (class III or IV according to the New York Heart Association classification); hemodynamically significant valvular heart disease; chronic infections; auto-inflammatory or autoimmune disease; cancer; therapy with steroids, immunosuppressive agents and non-steroidal anti-inflammatory drugs (excluding low doses of aspirin).

All patients gave their informed consent and the study was approved by the hospital's ethics committee.

\section{Laboratory measurements}

Peripheral venous blood was drawn into blood collection tubes in a standardized manner. All blood samples were collected under minimal tourniquet pressure from the antecubital vein using a wide caliber puncture needle (21-gauge). All blood tubes were immediately transferred to the 
Table I. Demographic and clinical characteristics of the study population

\begin{tabular}{|c|c|c|c|}
\hline Parameter & No HFrEF & HFrEF & $P$-value \\
\hline$N$ & 38 & 43 & \\
\hline Age [years] & 62.3 & 65.2 & 0.20 \\
\hline Sex (male \%) & 68 & 69 & \\
\hline Current smoking (\%) & $16(42)$ & $16(37)$ & 0.55 \\
\hline Hypertension (\%) & $23(61)$ & $33(77)$ & 0.12 \\
\hline Diabetes mellitus (\%) & $7(18)$ & $15(35)$ & 0.10 \\
\hline Family history (\%) & $14(37)$ & $5(12)$ & 0.008 \\
\hline ASS (\%) & $38(100)$ & $41(95)$ & 0.19 \\
\hline Clopidogrel (\%) & $37(97)$ & $40(93)$ & 0.38 \\
\hline$\beta$-blockers (\%) & $38(100)$ & $40(93)$ & 0.10 \\
\hline ACE-inhibitors (\%) & $36(95)$ & $38(88)$ & 0.32 \\
\hline AT1-blockers (\%) & $1(3)$ & $3(7)$ & 0.38 \\
\hline Statins (\%) & $37(97)$ & $40(93)$ & 0.11 \\
\hline Diuretics (\%) & $6(16)$ & $14(32)$ & 0.08 \\
\hline Calcium-channel blockers (\%) & $4(11)$ & $4(9)$ & 0.86 \\
\hline Coumadin (\%) & $1(3)$ & $5(12)$ & 0.13 \\
\hline LVEF (\%) & 51.1 & 31.9 & $<0.001$ \\
\hline CK peak $[\mathrm{U} / \mathrm{I}]$ & 2077.3 & 3537.2 & 0.004 \\
\hline CRP peak [mg/l] & 38.7 & 84.3 & $<0.001$ \\
\hline Creatinine $[\mathrm{mg} / \mathrm{l}]$ & 1.03 & 1.14 & 0.37 \\
\hline $\mathrm{BUN}[\mathrm{mg} / \mathrm{l}]$ & 29.8 & 40.90 & 0.03 \\
\hline Triglycerides & 135.1 & 129.7 & 0.51 \\
\hline Total cholesterol [mg/dl] & 224.7 & 201.1 & 0.06 \\
\hline HDL cholesterol & 50.3 & 47.9 & 0.41 \\
\hline LDL cholesterol & 148.5 & 133.1 & 0.11 \\
\hline $\mathrm{WBC}\left[10^{3} / \mu \mathrm{l}\right]$ & 11.2 & 12.7 & 0.50 \\
\hline Hemoglobin $[\mathrm{g} / \mathrm{dl}]$ & 14.6 & 14.1 & 0.22 \\
\hline Platelets $\left[10^{3} / \mu \mathrm{l}\right]$ & 266.3 & 255.6 & 0.55 \\
\hline Potassium [mmol/l] & 4.2 & 4.5 & 0.07 \\
\hline Sodium $[\mathrm{mmol} / \mathrm{l}]$ & 138.7 & 138.2 & 0.32 \\
\hline
\end{tabular}

laboratory. C-reactive protein and routine laboratory tests including serum electrolytes, total cholesterol (TC), high density lipoprotein (HDL), TC/ $\mathrm{HDL}$ ratio, triglycerides (TG), creatinine kinase $(C K)$, creatinine, white blood cells (WBC), hemoglobin and platelets were determined by standard laboratory methods (Table I).

C-reactive protein levels and CK activity were measured on admission, and after $6,12,24,30$, 48, 72 and $96 \mathrm{~h}$.

\section{Echocardiography}

To evaluate LVEF a 2-dimensional echocardiogram was performed on the first and seventh day after admission.

Standard transthoracic echocardiography was performed according to the recommendations of the European Association of Echocardiography on a Vivid 7 Dimension machine (GE Vingmed, Horton, Norway; M3S 2.5-MHz transducer). Images were stored digitally and analyzed off-line 
(EchoPac PC, GE Vingmed). For all measurements 3 beats were stored and analyzed. Left atrial and ventricular volumes, left ventricular (LV) end-diastolic diameter, and LV mass were calculated according to current recommendations and indexed for body surface area. Pulse-wave Doppler was performed in the apical 4-chamber view to obtain peak early filling (E wave) and late diastolic filling (A wave) velocities, E/A ratio, deceleration time of the early filling wave, and the isovolumic relaxation time. The frame rate for tissue Doppler imaging measurements was 100 frames/s. Timings of aortic valve opening, peak systolic contraction, and aortic valve closure were measured in relation to the beginning of the QRS complex.

\section{Evaluation of systolic heart failure}

The presence and degree of HF were assessed on the first and seventh day after admission following myocardial infarction. Heart failure was classically defined as the clinical diagnosis of $\mathrm{HF}$ and an $E F \leq 40 \%$, which is the official diagnosis of heart failure with reduced EF (HFrEF) [20, 21] (Table II).

\section{Follow-up}

Six months and one year after recruitment each patient was called for a clinical check-up. For those who died during a hospital stay, date and cause of death were obtained from hospital records (including the postmortem report where available). Mortality data for those who died outside the hospital were obtained from the family doctor. No patient dropped out during follow-up. End points used for the present study were global mortality and HF mortality.

\section{Statistical analysis}

Statistical analyses were performed with SPSS (Statistical Package for the Social Sciences) version 17.0. Categorical or dichotomous variables are expressed as absolute values and percentages, and were compared with Pearson's $\chi^{2}$ test. Continuous variables with a normal distribution are expressed as mean \pm standard deviation, and Student's $t$ test was used for comparisons between groups. Continuous variables without normal distribution are expressed as the median and interquartile range. Pearson's test was used to study the correlation between quantitative variables. A multivariate logistic regression model was used to evaluate the independent contribution of CRP levels to the risk of new events during follow-up. Univariate predictors of potential significance and CRP values were included in multivariate analysis. Adjusted hazard ratios and $95 \%$ confidence intervals $(\mathrm{Cl})$ are pre- sented. Kaplan-Meier curves were constructed to evaluate the prognostic value of CRP. A $p$-value $<0.05$ was considered statistically significant.

\section{Results}

Study patients had a mean \pm SD age of 63.8 \pm 11.9 years; $69 \%$ were male. All patients presented with STEMI in the Department of Cardiology, University Clinic of Erlangen. Fifty-one percent of the study patients presented with anterior, $48 \%$ with inferior and $1 \%$ with posterolateral STEMI. Forty-three patients developed significant HFrEF. All patients underwent acute cardiac catheterization. Patient characteristics are shown in Tables I and II. There were no significant peri- or post-procedural complications. Two patients needed catecholamines during the procedure. One patient had to be defibrillated due to ventricular fibrillation. Two patients developed acute renal failure, but without need of hemofiltration.

Table II. Procedural details of the study population

\begin{tabular}{|c|c|c|}
\hline Parameter & $\begin{array}{c}\text { No } \\
\text { HFrEF }\end{array}$ & HFrEF \\
\hline$N$ & 38 & 43 \\
\hline \multicolumn{3}{|l|}{ Lesion localization, $n$ (\%): } \\
\hline LAD & $22(58)$ & $19(44)$ \\
\hline LCX & $0(0)$ & $1(2)$ \\
\hline RCA & $23(61)$ & $16(37)$ \\
\hline \multicolumn{3}{|l|}{ Degree of CAD, $n(\%)$ : } \\
\hline One vessel & $10(26)$ & $23(53)$ \\
\hline Two vessels & $15(39)$ & $11(26)$ \\
\hline Three vessels & $10(26)$ & $12(28)$ \\
\hline \multicolumn{3}{|c|}{ TIMI flow target vessel pre PCI, $n(\%)$ : } \\
\hline 0 & $27(71)$ & $28(65)$ \\
\hline 1 & $5(13)$ & $10(23)$ \\
\hline 2 & $4(11)$ & $5(12)$ \\
\hline 3 & $0(0)$ & $2(5)$ \\
\hline \multicolumn{3}{|c|}{ TIMI flow target vessel post PCI, $n(\%)$ : } \\
\hline 0 & $0(0)$ & $0(0)$ \\
\hline 1 & $1(3)$ & $1(2)$ \\
\hline 2 & $3(8)$ & $2(5)$ \\
\hline 3 & $36(95)$ & $38(88)$ \\
\hline Mean number of stents per lesion & 1.09 & 1.13 \\
\hline \multicolumn{3}{|c|}{ Post-procedural complications, $n(\%)$ : } \\
\hline Acute renal insufficiency & $2(5)$ & $0(0)$ \\
\hline Bleeding & $0(0)$ & $1(2)$ \\
\hline Defibrillation & $1(3)$ & $0(0)$ \\
\hline
\end{tabular}


C-reactive protein levels were measured on admission and after 6, 12, 24, 30, 48, 72 and 96 h. The time course of CRP levels is shown in Figure 1. On admission CRP levels were already elevated in $92 \%$ of the patients $(15.1 \pm 27.7 \mathrm{mg} / \mathrm{l})$. C-reactive protein levels reached a peak after about $48 \mathrm{~h}$ $(59.2 \pm 59.6 \mathrm{mg} / \mathrm{l})$. Patients with STEMI and signs of heart failure showed significantly higher peak CRP levels than STEMI patients without signs of heart failure (Figure 2; median: $15.0 \mathrm{mg} / \mathrm{l} ; 25^{\text {th }}$ percentile: $7.5 \mathrm{mg} / \mathrm{l} ; 75^{\text {th }}$ percentile $41.0 \mathrm{mg} / \mathrm{l}$ vs. median: $61.5 \mathrm{mg} / \mathrm{l} ; 25^{\text {th }}$ percentile: $23.0 \mathrm{mg} / \mathrm{l} ; 75^{\text {th }}$ percentile $131.0 \mathrm{mg} / \mathrm{l}, p<0.001)$. Furthermore, we also found a significant negative correlation between LVEF and peak CRP levels (Figure $3 \mathrm{~A}$, $r=-0.56, p<0.001)$. When comparing maximum CK levels with peak CRP levels we also found a sig nificant positive correlation (Figure $3 \mathrm{~B}, r=0.52$, $p<0.001$ ).

Figure 4 shows the receiver operator characteristic curves (ROC) for CRP and CK for the prediction of heart failure following acute myocardial infarction. The area under the curve for CK was 0.41 (95\% Cl: $0.28-0.54)$ and CRP $0.76(95 \% \mathrm{Cl}$ :

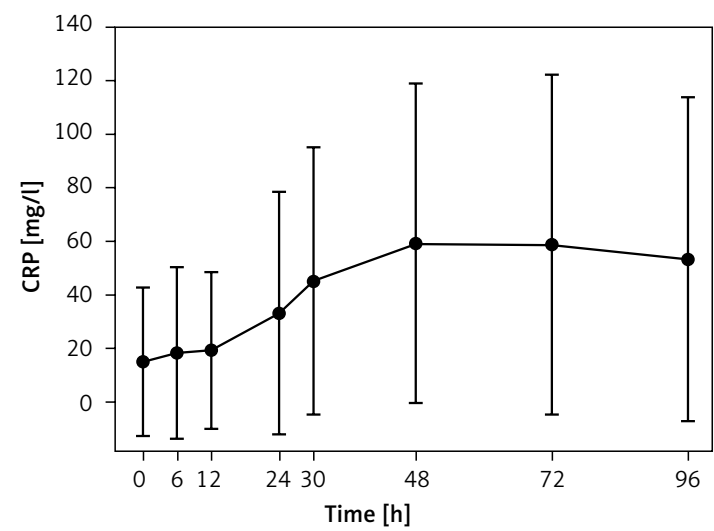

Figure 1. C-reactive protein (CRP) time course after ST-elevation myocardial infarction. Values are means and error bars indicate SEM

A

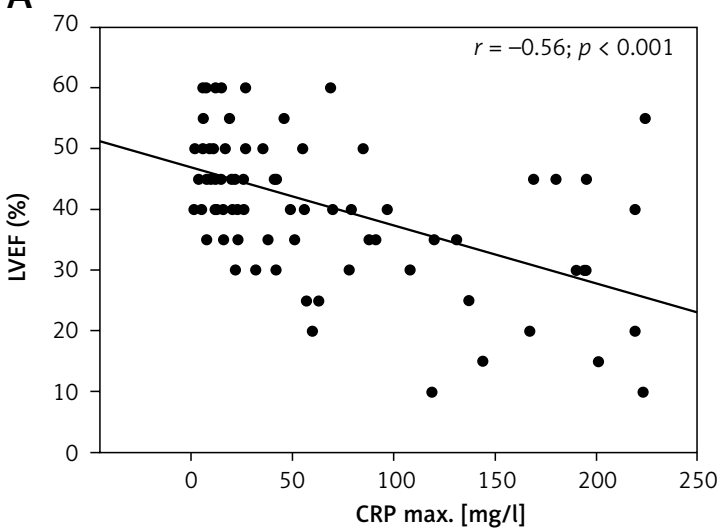

0.64-0.87) ( $p<0.001)$. The ROC analysis revealed an optimal cut-off point for peak CRP within the first $48 \mathrm{~h}$ following STEMI that identifies patients prone to HF progression and worse prognosis; it was $47.5 \mathrm{mg} / \mathrm{l}$.

None of the patients died during hospitalization. Patients with high CRP levels did not show significantly longer ICU stay or hospital stay. In the recruited patients no hemodynamic support devices had to be applied. During the follow-up of 12 months there were major adverse cardiovascular events in 19 (23\%) patients including 2 sudden deaths, 3 non-fatal AMI, and 2 patients underwent a coronary artery bypass graft (CABG) operation. Repeat percutaneous transluminal coronary angioplasty (PTCA) was performed in 12 patients. Five repeat PTCA procedures did not involve the study vessel and were motivated by progression of the disease in other vessels, whereas the other 6 were target lesion revascularization (TLR) procedures. Figure 5 shows the Kaplan-Meier plot for MACE-free survival within 12 months in patients with CRP levels above and below $47.5 \mathrm{mg} / \mathrm{l}$. Patients with CRP levels below 47.5 had significantly

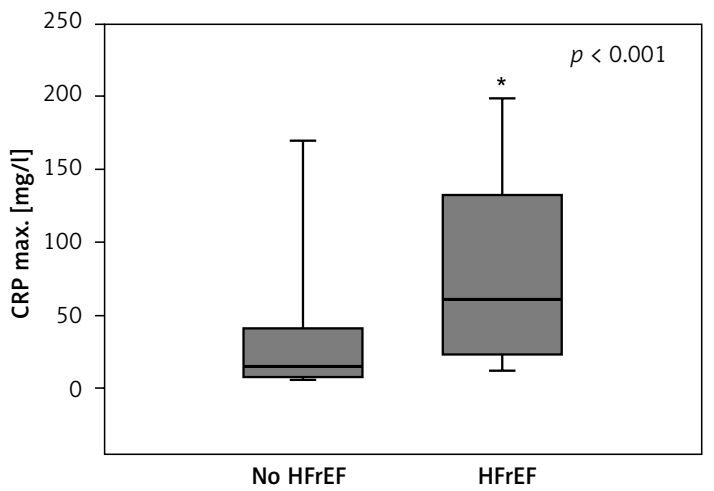

Figure 2. C-reactive protein (CRP) levels of ST-elevation myocardial infarction patients with and without heart failure. Solid lines indicate median values and boxes indicate $25-75^{\text {th }}$ percentiles

B

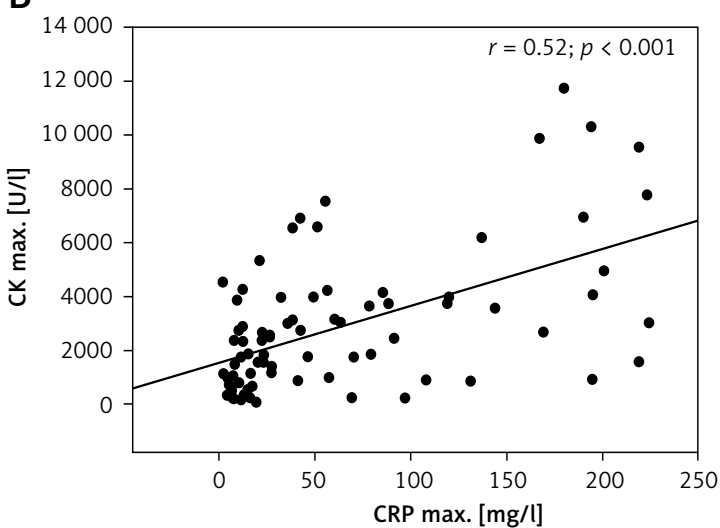

Figure 3. A - Negative correlation between C-reactive protein (CRP) level and left ventricular ejection fraction (LVEF). B - Positive correlation between CRP level and creatinine kinase (CK) activity $(r=0.52, p<0.001)$ 


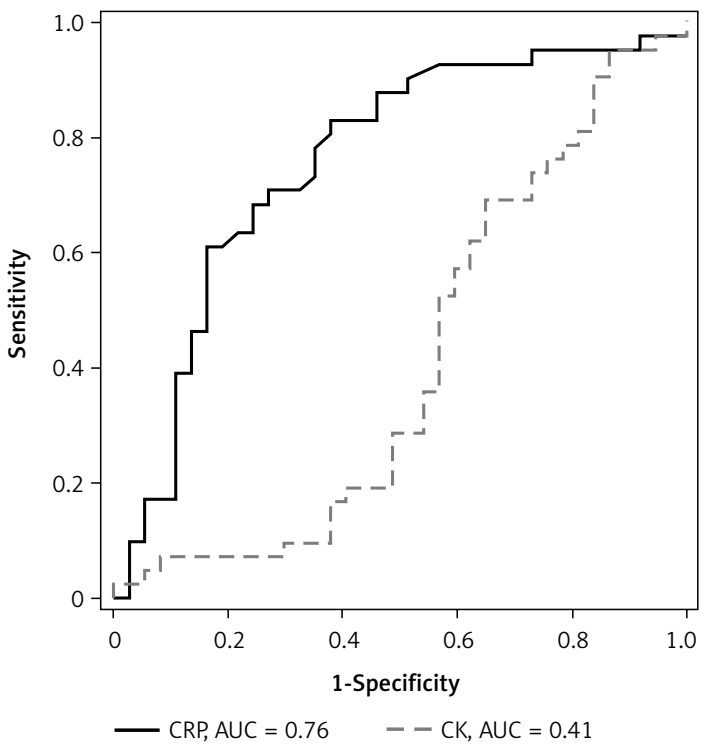

Figure 4. Receiver operating characteristic curves for the prediction of heart failure after ST-elevation myocardial infarction. The area under the curve (AUC) was calculated as 0.76 for C-reactive protein (CRP); 95\% confidence interval: $0.64-0.87$ and 0.41 for creatine kinase (CK) levels (95\% confidence interval: $0.28-0.54, p<0.001)$

fewer major cardiovascular adverse events than patients with higher CRP levels.

\section{Discussion}

Our study shows a clear relationship between in-hospital CRP plasma concentrations and the development of post-infarction heart failure in patients undergoing $\mathrm{PCl}$ for a first STEMI.

Our study is in agreement with other reports, where the acute inflammatory reaction in $\mathrm{AMI}$ starts 4 to $8 \mathrm{~h}$ after the acute onset and peaks within $48 \mathrm{~h}$ [22]. Furthermore, we showed that peak CRP levels identify patients prone to HF progression and worse prognosis. In a previous study Berton et al. found an association between CRP levels and heart failure in patients with AMI [23]. However, they examined patients with myocardial infarction in general and did not differentiate between STEMI and non-STEMI (NSTEMI). Furthermore, they included patients with previous myocardial infarction and some of the patients were treated with thrombolysis. In our study, we recruited only STEMI patients without a prior history of myocardial infarction. All of our patients were treated with primary $\mathrm{PCl}$ within 60 min after hospital admission.

Generally, CRP is an acute phase reactant, synthesized primarily by the liver. For many years CRP has thus been merely used as a marker for systemic inflammation. However, there are accumulating data indicating that CRP could be more than just a biomarker of inflammation.

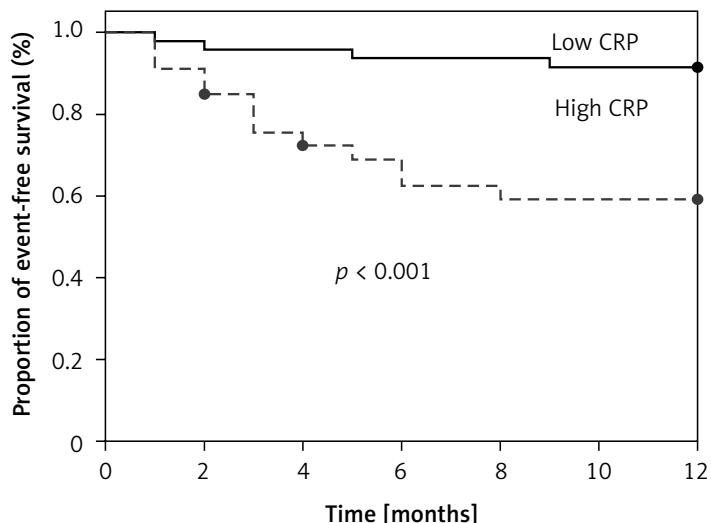

Figure 5. Kaplan-Meier estimates of major adverse cardiac events (MACE)-free survival within 12 months in patients with $\mathrm{C}$-reactive protein (CRP) levels above or below $47.5 \mathrm{mg} / \mathrm{l}$. The MACE includes cardiac death, myocardial infarction, or target lesion revascularization $(p<0.001)$

Previous data indicate that CRP may induce complement activation and thus may lead to maladaptive cardiac remodeling in acute ischemic left ventricular damage. After aggregation and ligand binding, CRP is a potent activator of the classical complement pathway. In this context, CRP has been found on myocardial cells within the infarcted area together with activated complement [12]. In diverse animal models, several authors have shown that CRP markedly increases the extent of myocardial damage produced by ischemic injury [13, 14, 24].

In our study, the rapid rise in CRP levels reflects the severity of the inflammatory reaction within the infarcted area. The CRP levels positively correlated with CK activity and negatively with left ventricular ejection fraction. The extent of inflammatory activation seems to be a major determinant in post-infarction remodeling. Combining the respective preclinical data $[13,14]$ with our results, high CRP levels may not only be a sign of an activated inflammatory reaction, but also a determinant of further myocardial damage. After aggregation or ligand binding, human CRP activates the classical complement pathway [25, 26]. In this concept, CRP also binds to non-irremediable damaged cells and leads to complement activation. As a consequence, the targeted cells were opsonized, thereby increasing the amount of cell death and thus increasing infarct size. This concept could in part explain our results that patients with high CRP levels developed post-infarction heart failure. Large infarct size, thus resulting in heart failure, is furthermore a major determinant of morbidity and mortality following STEMI. In our patients stratified by CRP levels, the cut-off value was $47.5 \mathrm{mg} / \mathrm{l}$ for predicting heart failure and cardiovascular adverse events within the first 12 months following STEMI. Together with the 
data from other groups the marked rise in patients developing heart failure may not only be an epiphenomenon but may represent a pathogenic process that leads to further myocardial damage and thus heart failure.

Generally, inflammation plays a very important role in wound healing after myocardial infarction. Since CRP is one of the acute phase proteins, it is very important in "orchestrating" one of the first inflammatory responses. An excessive inflammatory response, however, leads to worsening heart failure and poor outcome after myocardial infarction.

Therapeutic inhibition of an excessive CRP response could thus be a promising new approach to cardioprotection in STEMI patients. In this context Pepys et al. developed a specific small-molecule inhibitor of CRP [14]. In rats undergoing AMI this inhibitor could abrogate the increase in infarct size and cardiac dysfunction. In another interesting approach Sheriff et al. developed a specific CRP adsorber and showed that CRP depletion by apheresis led to a reduction of infarct size in a porcine animal model of AMI [15]. Although it is difficult to transfer these data to the clinical setting of patients suffering STEMI, it supports the assumption that therapeutic inhibition of CRP could be a promising approach to cardioprotection after STEMI. However, further investigations need to be performed to test the impact of therapeutic CRP depletion in a human clinical setting.

Although there is no widespread use of the only approved anti-CRP treatment possibility (CRP apheresis) at the moment, our data may make an important contribution to identify STEMI patients at risk for heart failure and worse prognosis. Once identified, these patients could perhaps benefit from a specific heart failure therapy or may furthermore be evaluated for strict monitoring up to the use of a LifeVest defibrillator for the first weeks after acute STEMI. However, further studies are needed to confirm these data in a large trial.

\section{Conflict of interest}

The authors declare no conflict of interest.

\section{References}

1. Ross R. Atherosclerosis: an inflammatory disease. N Engl J Med 1999; 340: 115-26.

2. Koyama K, Yoneyama K, Mitarai T, et al. Association between inflammatory biomarkers and thin-cap fibroatheroma detected by optical coherence tomography in patients with coronary heart disease. Arch Med Sci 2015; 11: 505-12.

3. Mrowiecki W, Sagan A, Guzik B, et al. Inflammatory aortic abdominal aneurysm - immunophenotypic characterization of inflammatory infiltrate. Arch Med Sci 2014; 10: $1258-62$

4. Tiyerili V, Becher UM, Camara B, et al. Impact of peroxisome proliferator-activated receptor gamma on an- giotensin II type 1 receptor-mediated insulin sensitivity, vascular inflammation and atherogenesis in hypercholesterolemic mice. Arch Med Sci 2015; 11: 877-85.

5. Dibra A, Mehilli J, Schwaiger $M$ et al. Predictive value of basal C-reactive protein levels for myocardial salvage in patients with acute myocardial infarction is dependent on the type of reperfusion treatment. Eur Heart J 2003; 24: 1128-33.

6. Bisoendial RJ, Kastelein JJ, Stroes ES. C-reactive protein and atherogenesis: from fatty streak to clinical event. Atherosclerosis 2007; 195: e10-8.

7. Pearson TA, Mensah GA, Alexander RW, et al. Markers of inflammation and cardiovascular disease: application to clinical and public health practice. A statement for healthcare professionals from the Centers for Disease Control and Prevention and the American Heart Association. Circulation 2003; 107: 499-511.

8. Xie D, Hu D, Zhang O, Sun Y, Li J, Zhang Y. Increased high-sensitivity C-reactive protein, erythrocyte sedimentation rate and lactic acid in stroke patients with internal carotid artery occlusion. Arch Med Sci 2016; 12: 546-51.

9. Ridker PM, Cushman M, Stampfer MJ, Tracy RP, Hennekens $\mathrm{CH}$. Inflammation, aspirin, and the risk of cardiovascular disease in apparently healthy men. N Engl J Med 1997; 336: 973-9.

10. Danesh J, Wheeler JG, Hirschfield GM, et al. C-reactive protein and other circulating markers of inflammation in the prediction of coronary heart disease. N Engl J Med 2004; 350: 1387-97.

11. Kroop IG, Shackman NH. Level of C-reactive protein as a measure of acute myocardial infarction. Proc Soc Exp Biol Med 1954; 86: 95-7.

12. Lagrand WK, Niessen HW, Wolbink GJ, et al. C-reactive protein colocalizes with complement in human hearts during acute myocardial infarction. Circulation 1997; 95: 97-103.

13. Griselli M, Herbert J, Hutchinson WL, et al. C-reactive protein and complement are important mediators of tissue damage in acute myocardial infarction. J Exp Med 1999; 190: 1733-40.

14. Pepys MB, Hirschfield GM, Tennent GA, et al. Targeting C-reactive protein for the treatment of cardiovascular disease. Nature 2006; 440: 1217-21.

15. Sheriff A, Schindler R, Vogt B, et al. Selective apheresis of $C$-reactive protein: a new therapeutic option in myocardial infarction? J Clin Apher 2015; 30: 15-21.

16. Pasceri V, Willerson JT, Yeh ET. Direct proinflammatory effect of C-reactive protein on human endothelial cells. Circulation 2000; 102: 2165-8.

17. Heinecke JW. Chemical knockout of C-reactive protein in cardiovascular disease. Nat Chem Biol 2006; 2: 300-1.

18. Emanuelsson $\mathrm{H}$, Karlson BW, Herlitz J. Characteristics and prognosis of patients with acute myocardial infarction in relation to occurrence of congestive heart failure. Eur Heart J 1994; 15: 761-8.

19. Pietila KO, Harmoinen AP, Jokiniitty J, Pasternack Al. Serum C-reactive protein concentration in acute myocardial infarction and its relationship to mortality during 24 months of follow-up in patients under thrombolytic treatment. Eur Heart J 1996; 17: 1345-9.

20. Yancy CW, Jessup M, Bozkurt B, et al. 2013 ACCF/AHA guideline for the management of heart failure: a report of the American College of Cardiology Foundation/ American Heart Association Task Force on Practice Guidelines. J Am Coll Cardiol 2013; 62: e147-239.

21. Ponikowski P, Voors AA, Anker SD, et al. 2016 ESC Guidelines for the Diagnosis and Treatment of Acute and 
Chronic Heart Failure. Rev Esp Cardiol (Engl Ed) 2016; 69: 1167.

22. Beranek JT. C-reactive protein and complement in myocardial infarction and postinfarction heart failure. Eur Heart J 1997; 18: 1834-6.

23. Berton G, Cordiano R, Palmieri R, Pianca S, Pagliara V, Palatini P. C-reactive protein in acute myocardial infarction: association with heart failure. Am Heart J 2003; 145: 1094-101.

24. Barrett TD, Hennan JK, Marks RM, Lucchesi BR. C-reactive-protein-associated increase in myocardial infarct size after ischemia/reperfusion. J Pharmacol Exp Ther 2002; 303: 1007-13.

25. Volanakis JE. Complement activation by C-reactive protein complexes. Ann N Y Acad Sci 1982; 389: 235-50.

26. Jiang H, Robey FA, Gewurz H. Localization of sites through which C-reactive protein binds and activates complement to residues 14-26 and 76-92 of the human C1q A chain. J Exp Med 1992; 175: 1373-9. 